\title{
Research on Fusion Technology of Fuzzy System and Neural Network
}

\author{
Li Yang \\ Nanchang Institute of Science \& Technology, Nanchang, Jiangxi, 330108
}

\begin{abstract}
The application of fuzzy reasoning and neural network in information fusion has its own merits. In contrast, the advantage of fuzzy reasoning lies in its strong ability of knowledge expression, its internal model structure and reasoning results are easy to understand, the disadvantage is that learning is limited, knowledge acquisition is difficult; Learning ability, the disadvantage is that neural network in the training process to obtain knowledge is a "hidden" way stored in the network, the model for the network structure is difficult to give a reasonable explanation. To this end, the combination of the two, avoid weaknesses, both for information fusion technology the only way.
\end{abstract}

Keywords: fusion technology, fuzzy system, neural network

\section{Introduction}

Information fusion, here mainly refers to the multi-sensor information fusion, is the different knowledge sources and multiple sensors to obtain the information integrated processing, eliminating multi-sensor information between the possible redundancy and contradictions, the use of information complementarity, reduce uncertainty To form a relatively complete understanding of the system environment, thereby improving the intelligent system decision-making, scientific planning, rapid response and correctness, and thus reduce the risk of decision-making process. Since the information fusion technology has been put forward, it is increasingly subject to scientists and government departments of the military attention. In order to quickly occupy the commanding heights of this key technology, countries have invested a lot of manpower and material resources for 
the development of this emerging intelligent technology. At the same time, the information fusion technology academic papers, academic conferences and research projects are also increasing, the research method of information fusion is endless. According to incomplete statistics, information fusion methods have reached as many as dozens. Information fusion technology is the first application in the military developed, with the various fields of experts and scholars in-depth study of the civil aspects of the application has also been developed by leaps and bounds. In the military field, the main application is the detection or monitoring and diagnosis system, intelligence (defense, target acquisition) and strategic early warning defense system; the main application of a maritime surveillance system, air-to-air or ground-to-ground defense system, Robotics, intelligent manufacturing systems, modern management and multimedia systems.

\section{Information Fusion Technology of Neural Network}

Artificial neural network, referred to as neural network, is a large number of artificial neurons in accordance with a certain topology of the formation of extensive interconnection, and in accordance with certain learning rules, through a large number of sample data learning and training, the network " "Stored in the form of connection weights and thresholds between neurons, and the use of these" knowledge "to achieve a certain human brain function inference engine. To this end, the characteristics and functions of a neural network depends on three elements: one is the basic unit of neurons; neurons; the connection mode between neurons; the topological structure of neural networks; Neural network learning and training, to amend the connection between neurons weights and threshold learning rules.

Neural networks are mainly composed of neurons. In artificial neural networks, neurons are often referred to as "processing units." Sometimes referred to as a "node" from the network point of view. Artificial neuron is a formal description of biological neurons, which abstracts the information processing of biological neurons and describes them in mathematical language. The structure and function of biological neurons are simulated and expressed by model graphs . At present, there are many neuron models proposed, among which the earliest proposed and the most influential are the M-P model first proposed by the psychologist McCulloch and mathematician W. Pitts in 1943 to analyze and summarize the basic characteristics of neurons. The model is continually improved to form a widely used formal neuron model.

From the viewpoint of information fusion, neural network is a very large-scale parallel information fusion processing system with high nonlinearity. It can be regarded as a fusion system to realize some function transformation of multiinput signals. In the multi-sensor system, the environmental information provided by each information source has a certain degree of uncertainty, the fusion process of these uncertain information is essentially a process of uncertainty reasoning. The neural network can determine the classification criteria according to the similarity of the samples received by the current system. This method is mainly 
expressed in the weight distribution of the network. At the same time, neural network specific learning algorithm can be used to obtain knowledge, mechanism. The basic idea of information fusion with neural network is to simulate the learning of human brain, associative memory and the integration of information and other functions. The neural network system uses the adaptive and selflearning ability of the neural network to store a large amount of sensor information (sample data) in the form of network connection weights and thresholds in the network. The new uncertainty information of the system makes the uncertainty reasoning, which is to simulate the human brain's learning ability. With the help of neural network's unique associative memory function, the sensor information can be restored when some sensors are faulty or error. Neural network system uses powerful non-linear mapping function to correlate and integrate the information obtained by the sensor to realize the nonlinear mapping between the input and output of the system and ensure that the different input can obtain the corresponding Of the correct output, which is the integration of human brain information simulation.

\section{Information fusion technology based on fuzzy reasoning}

It should be noted that the determination of the membership function is essentially a qualitative description of the transitions of the intermediary of objective things, which are essentially objective. For the same fuzzy concept, different people will not create exactly the same membership function. So, theoretically, even if the membership function determined according to expert experience, this method is not theorized can not guarantee its correctness, because any person's knowledge and experience are limited. However, in practical applications, although different methods to determine the membership function, in a certain range, there may be some nuances, but in the end can achieve the desired goal. In other words, the determination of membership functions is not unique, allowing different combinations. Therefore, in order to simplify the calculation, many membership function curves are triangles. In fact, according to the fuzzy statistical method to get the membership function is usually bell-shaped, so the triangle membership function is not the best function, but an approximation. Computer simulation experiments show that the shape of the membership function can affect the whole process of the fuzzy system, for example, it will affect the time of the fuzzification and de-fuzzification of the MCU and the storage space of the query table (now commonly used triangle, trapezium and Single-line shape (also known as bar), because the practice has proved that it can meet the general requirements, but also simplify the calculation, it is widely used.

Fuzzy reasoning is an approximate simulation of the logic reasoning ability of human brain. It first uses fuzzy sets and membership functions to describe the fuzziness and uncertainty of things and phenomena in real life. Fuzzy rules are established by the knowledge of experts in various fields. Fuzzy reasoning is a kind of uncertainty approximation reasoning, so fuzzy reasoning is a kind of 
uncertainty reasoning. In this way, fuzzy reasoning is a kind of fuzzy inference system. . The basic idea of information fusion using fuzzy reasoning is to simulate the process of human brain dealing with uncertainty information. First, fuzzy sets and membership functions are used to describe the fuzzy information obtained by multi-sensors. Then, different fuzzy rules are established according to experts' knowledge in different fields. Finally, the fuzzy reasoning is carried out by using various fuzzy logic operators, and the multi-sensor information is processed synthetically in the course of reasoning, and the final result of information fusion is deduced.

(1) sensor selection. According to the specific practical problems, select the appropriate sensor for detection, access to practical problems in the study of the relevant information (including data and signals, etc.).

(2) The design of fuzzy inference system, including fuzzy set membership function and fuzzy inference rules. According to the method of determining the membership functions described in the previous section, fuzzy sets and membership functions are used to describe the sensor information variables. For different problems, the fuzzy inference rules are determined by the knowledge and experience of experts in different fields.

(3) Fuzzy reasoning. The fuzzy logic operator is used to compute the conclusion of each rule, and each conclusion represents the degree of satisfaction of the rule.

(4) Fuzzy synthesis. After fuzzy reasoning, every fuzzy rule is deduced and a fuzzy conclusion is derived. Then, the fuzzy conclusion is realized by fuzzy synthesis, and a general conclusion is drawn.

(5) anti-fuzzy. In order to facilitate the practical application, through a variety of anti-fuzzy process, the fuzzy inference fusion results - a fuzzy set membership function into an accurate value.

\section{The main Combination way of neural network and fuzzy reasoning}

In a simple way, the fuzzy inference system and the neural network system exist in their own way and play a certain role. Mainly in the following three ways:

Loose type combination. In a system, the part that can be represented by the "ifthen" rule is described by the fuzzy inference system, while the part which is difficult to express by the "if-then" rule is denoted by the neural network, contact directly;

Fuzzy reasoning and neural networks are connected in parallel in the system, ie, they share a common input. Fuzzy inference and neural network are connected in series in the system, that is, one output is the other input, which can be regarded as the preprocessing part of the two input signals. For example, the neural network preprocessing the input data of the fuzzy inference system can realize data collation, aggregation and noise suppression, and make the process of fuzzy rules easy. 
The key to the design of the fuzzy reasoning system is the acquisition of knowledge. It is difficult to acquire the rules and adjust the membership function effectively. It is difficult to realize the self-learning function. In the fuzzy system enhanced by neural network, the learning ability of the neural network can overcome these problems.

\section{Conclusion}

Fuzzy reasoning is a simple simulation of the logical reasoning ability of a person. It can deal with fuzzy information well, and with the human language similar to the fuzzy language and statements to describe the real world and a variety of phenomena, have a strong knowledge of expression. Through the analysis of the knowledge and experience of experts in related fields, these knowledge and experience are described by fuzzy language. Different things and phenomena are usually described in the form of fuzzy sets and membership functions, and fuzzy rules (statements) are used to describe things and phenomena, And the fusion of the input information of the system is realized by fuzzy reasoning (a certain fuzzy operation), and the fusion result is obtained. In fact, this process is a simple simulation of the practical problems of experts, reflecting the wisdom of mankind.

\section{References}

[1] Peng Yu. Temperature Control of Microwave Oven Based on Adaptive Neuro-Fuzzy Inference System. Journal of Shaanxi University of Science and Technology. , 55(12), pp.68-70, 2011

[2] Chen HuiPing. Nonlinear Control Based on Adaptive Neuro-Fuzzy Inference System .Computer Simulation, , 8 (5), pp.87- 91, 2013

[3] Li Hongsheng. Application of ANFIS fuzzy neural inference machine in fault diagnosis .Control Engineering, , 12(10), pp. 18- 20, 2012

[4] Wu Xiaoqun. An Information Fusion Method Based on Fuzzy Neural Network. Journal of Shanxi Finance and Economics University, 9(6), pp.58- 61, 2011

[5] Zhou Chaochao, Zhang Yongzhi, Cheng Shengwen. Application of neural network in multi-sensor information integration and fusion. 8(4), pp.85-87, 2014 